\title{
ALL TRIANGLES ARE RAMSEY
}

\author{
PETER FRANKL AND VOJTECH RÖDL
}

\begin{abstract}
Given a triangle $A B C$ and an integer $r, r \geq 2$, it is shown that for $n$ sufficiently large and an arbitrary $r$-coloring of $R^{n}$ one can find a monochromatic copy of $A B C$.
\end{abstract}

I. Introduction. Let $R^{n}$ denote $n$-dimensional Euclidean space. A finite pointset $A=\left\{A_{1}, \ldots, A_{s}\right\}$ is said to be Ramsey if for every integer $r$ there exists an $n_{0}=n_{0}(A, r)$, so that if the points of $R^{n}$ are partitioned into $r$ classes one can always find a congruent copy of $A$ inside one class. In [1] it is proved that the vertex set (and consequently each subset) of a brick (rectangular parallelepiped) of arbitrary dimension is Ramsey. On the other hand, it is shown there that if $A$ is Ramsey, then it is spherical; i.e., it is contained in a sphere.

The first open question which arises is to decide whether obtuse triangles are Ramsey.

\section{THEOREM 1. All triangles are Ramsey.}

For the proof of this result we use two well-known results.

RAMSEY'S THEOREM [2]. Given integers $k, l, r \geq 2$ there exists $n_{0}=n_{0}(k, l, r)$ so that for all $n \geq n_{0}$ and all $r$-colorings of the l-subsets of $\{1,2, \ldots, n\}$ one can find a $k$-element set all of whose l-subsets have the same color.

Suppose $\mathbf{A}, \mathbf{B}$ are finite point sets in $R^{d}, R^{s}$, respectively. Define $\mathbf{A} * \mathbf{B}=$ $\{A * B: A \in \mathbf{A}, B \in \mathbf{B}\}$, where for $A=\left(a_{1}, \ldots, a_{d}\right), B=\left(b_{1}, \ldots, b_{s}\right), A * B=$ $\left(a_{1}, \ldots, a_{d}, b_{1}, \ldots, b_{s}\right)$ is a print in $R^{d+s}$.

Product theorem [1]. If $\mathbf{A}, \mathbf{B}$ are Ramsey, then $A * B$ is Ramsey too.

PROOF OF THE THEOREM. We prove the theorem in three stages.

Stage 1. The triangle with side lengths $\sqrt{2 t}, \sqrt{2 t}, \sqrt{8 t-6}$ is Ramsey for all $t \geq 2$.

Choose $n=n_{0}(2 t+1,2 t-1, r)$ from Ramsey's Theorem. Let us associate with each $(2 t-1)$-subset $B=\left\{i_{1}, \ldots, i_{2 t-1}\right\}$, where $1 \leq i_{1}<i_{2}<\cdots<i_{2 t-1} \leq n$, a point $P(B)=\left(x_{1}, \ldots, x_{n}\right)$ by setting $x_{j}=0$ unless $j \in B, x_{i_{\nu}}=\nu$ for $\nu=1, \ldots, t$ and $x_{i_{\nu}}=2 t-\nu$ for $\nu=t+1, \ldots, 2 t-1$. For example, for $B=\{1,4,6\}$ and $n=6$ we have $P(B)=\{1,0,0,2,0,1\}$.

Suppose the points of $R^{n}$, and, in particular, the points of form $P(B)$ are $r$ colored. This defines an $r$-coloring of the $(2 t-1)$-subsets of $\{1,2, \ldots, n\}$. By Ramsey's Theorem there exists a set $A=\left\{j_{1}, \ldots, j_{2 t+1}\right\}$ all of whose $(2 t-1)$ subsets have the same color.

Received by the editors June 5, 1985 and, in revised form, September 10, 1985.

1980 Mathematics Subject Classification (1985 Revision). Primary 05AXX. 
Choosing $A_{1}=\left\{j_{1}, \ldots, j_{2 t-1}\right\}, A_{2}=\left\{j_{2}, j_{3}, \ldots, j_{2 t}\right\}$ and $A_{3}=\left\{j_{3}, \ldots, j_{2 t+1}\right\}$, $P\left(A_{1}\right), P\left(A_{2}\right)$ and $P\left(A_{3}\right)$ have the same color. An easy computation shows that the distances are

$$
d\left(P\left(A_{1}\right), P\left(A_{2}\right)\right)=d\left(P\left(A_{2}\right), P\left(A_{3}\right)\right)=\sqrt{2 t}, \quad d\left(P\left(A_{1}\right), P\left(A_{3}\right)\right)=\sqrt{8 t-6} .
$$

Note that for $t \rightarrow \infty$ the largest angle of this triangle tends to $180^{\circ}$.

Stage 2. All isosceles triangles are Ramsey.

Let $A B C$ be a triangle with side lengths $a, a, b, \overline{B C}=b$. Let the angle at $A$ be $\alpha$, and choose $t$ so large that the angle of $P\left(A_{1}\right) P\left(A_{2}\right) P\left(A_{3}\right)$ at $P\left(A_{2}\right)$ is larger than $\alpha$.

Let us now rotate $A B C$ around $B C$, and denote by $A(\beta)$ the orthogonal projection of $A$ on the plane after rotation by angle $\beta$. Since $A\left(90^{\circ}\right)$ is on $B C$, there exists a $\beta$ for which $A(\beta) B C$ is congruent to $P\left(A_{1}\right) P\left(A_{2}\right) P\left(A_{3}\right)$. By the product theorem the prism $\{A(\alpha), B, C\} *\{0, \overline{A A(\alpha)}\}$ is Ramsey. Since $A B C$ is embedded into this prism, it is Ramsey.

Before going to Stage 3 let us extract some slightly more general results by the methods of Stages 1 and 2.

Stage $2^{\prime}$. Suppose the triangle $A^{\prime} B C$ is obtained from $A B C$ by orthogonal projection on a plane through $B C$. If $A^{\prime} B C$ is Ramsey, then so is $A B C$. Note also that if $\beta^{\prime}$ and $\beta$ ( $\gamma^{\prime}$ and $\left.\gamma\right)$ are the angles at $B(C)$ in the two triangles, respectively, then $\tan \gamma / \tan \beta$ and $\tan \gamma^{\prime} / \tan \beta^{\prime}$ are equal.

This makes possible a reformulation of the above statement. If a triangle with angles $\alpha^{\prime}, \beta^{\prime}$ is Ramsey, $\alpha^{\prime}<\alpha$ and $\tan \alpha / \tan \beta=\tan \alpha^{\prime} / \tan \beta^{\prime}$, then any triangle with angles $\alpha, \beta$ is Ramsey.

Stage $1^{\prime}$. If $p, q$ are integers, then for an arbitrary $\varepsilon>0$ there exist triangles with angles $\alpha, \beta$ which are Ramsey and which satisfy $|\tan \alpha / \tan \beta-p / q|<\varepsilon, \alpha+\beta<\varepsilon$.

Choose this time $n=n_{0}(2 t+p+q-1,2 t-1, r)$ and proceed as in Stage 1. Find $\left\{j_{1}, j_{2}, \ldots, j_{2 t+p+q-1}\right\}$, all of whose $(2 t-1)$-subsets have the same color. Set $A_{1}=$ $\left\{j_{1}, \ldots, j_{2 t-1}\right\}, A_{2}=\left\{j_{p+1}, \ldots, j_{2 t+p-1}\right\}, A_{3}=\left\{j_{p+q+1}, \ldots, j_{2 t+p+q-1}\right\}$. Let $\alpha_{i}$ be the angle at $A_{i}$ in $A_{1} A_{2} A_{3}$. It is easy to see that, for $t \rightarrow \infty, \tan \alpha_{1} / \tan \alpha_{3} \rightarrow p / q$ and $\alpha_{1}+\alpha_{3} \rightarrow 0$.

Stage 3. All triangles are Ramsey.

Let $A B C$ be an arbitrary triangle with angles $\alpha, \beta, \gamma$. Suppose $\alpha \leq \beta \leq \gamma$ and rotate $A B C$ around $B C$ with $A(\delta)$ being the image of $A$ by the orthogonal projection as the inclination of $A B C$ is $\delta$. Let $\alpha(\delta), \beta(\delta)$ be the corresponding angles. Then

$$
\lim _{\delta \rightarrow 90^{\circ}} \frac{\tan \alpha(\delta)}{\tan \beta(\delta)}=\frac{\overline{C A\left(90^{\circ}\right)}}{\overline{B C}} \stackrel{\text { def }}{=} c .
$$

Let $p / q$ be a rational number between $c$ and $\tan \alpha / \tan \beta$. By a continuity argument one can choose $\delta$ so that $\tan \alpha(\delta) / \tan \beta(\delta)=\tan \alpha_{1} / \tan \alpha_{3}, \alpha_{1}<\alpha(\delta)$ and $\alpha_{2}, \alpha_{3}$ are angles from Stage $1^{\prime}$.

By Stage $2^{\prime}$ we infer that triangles with angles $\alpha(\delta), \beta(\delta)$ are Ramsey, and again by Stage $2^{\prime}$ it follows that those with angles $\alpha, \beta, \gamma$ are Ramsey too.

II. Concluding remarks. One can apply the approach of Stage 1 to obtain that many other finite configurations are Ramsey. However, the dimension of these configurations tends to infinity as the number of points increases. In fact, we 
were unable to prove the Ramseyness for any pentagon. We can show that some symmetric trapezoids are Ramsey. Here is the argument.

Let us associate with $\{i, j\} \subset\{1,2, \ldots, n\}, i<j$, the point $P(i, j)$ which has $i$ th coordinate $1, j$ th coordinate -2 and all others 0 .

If $n \geq n_{0}(4,2, r)$ and $R^{n}$ is colored by $r$ colors, as in Stage 1 , we can find $\left\{j_{1}, j_{2}, j_{3}, j_{4}\right\}$ so that all six 2 -subsets of it have the same color. This will give a monochromatic configuration isometric to the point set $(1,0,-2,0),(0,0,1,-2)$, $(1,-2,0,0),(0,1,0,-2)$. It is easy to check that these points are coplanar, and in fact they span a symmetric trapezoid with sides $\sqrt{10}, \sqrt{8}, \sqrt{10}, \sqrt{2}$ and diagonals of length $\sqrt{14}$.

Using the product theorem, one obtains an infinity of other symmetric trapezoids which are Ramsey.

We can also prove that all simplices in arbitrary dimensions are Ramsey. The proof, however, is less elementary. We shall return to this problem in a later paper.

\section{REFERENCES}

1. P. Erdös, R. L. Graham, P. Montgomery, B. L. Rothschild, J. H. Spencer and E. G. Straus, Euclidean Ramsey theorems, J. Combin. Theory Ser. A 14 (1973), 341-363.

2. F. P. Ramsey, On a problem of formal logic, Proc. London Math. Soc. 30 (1930), 264-286.

Département de Mathématiques, Université de Paris VII, CNRS, 2 Place JUSSIEU, 75005 PARIS, FRANCE

Department of Mathematics, FJfi CVUT, husova 5, 11000 Praha 1, CzechosloVAKIA 